\title{
Beta-alanine supplementation enhances judo-related performance in highly-trained athletes
}

\author{
Caroline de Andrade Kratz ${ }^{\mathrm{a}}$, Vitor de Salles Painelli ${ }^{\mathrm{a}, *}$, \\ Kleiner Márcio de Andrade Nemezio ${ }^{a}$, Rafael Pires da Silva ${ }^{a}$, Emerson Franchini ${ }^{\mathrm{b}}$, \\ Alessandro Moura Zagatto ${ }^{c}, B^{2}$ runo Gualano ${ }^{a}$, Guilherme Giannini Artioli ${ }^{a}$ \\ a Laboratory of Applied Nutrition and Metabolism, School of Physical Education and Sport, University of São Paulo, Brazil \\ ${ }^{b}$ Combat Sports and Martial Arts Research Group, School of Physical Education and Sport, University of São Paulo, Brazil \\ ${ }^{c}$ Laboratory of Physiology and Sport Performance (LAFIDE), Faculty of Sciences, Paulista State University, Brazil
}

\section{A R T I C L E I N F O}

\section{Article history:}

Received 25 February 2016

Received in revised form 27 July 2016

Accepted 19 August 2016

Available online 26 August 2016

\section{Keywords:}

Carnosine

Buffers

Athletes

Dietary supplement

\begin{abstract}
A B S T R A C T
Objectives: In official judo competitions, athletes usually engage in 5-7 matches in the same day, performing numerous high-intensity efforts interspersed by short recovery intervals. Thus, glycolytic demand in judo is high and acidosis may limit performance. Carnosine is a relevant intracellular acid buffer whose content is increased with beta-alanine supplementation. Thus, we hypothesized that beta-alanine supplementation could attenuate acidosis and improve judo performance.

Design: Twenty-three highly-trained judo athletes were randomly assigned to receive either beta-alanine $\left(6.4 \mathrm{~g} \mathrm{day}^{-1}\right)$ or placebo (dextrose, same dosage) for 4 weeks.

Methods: Performance was assessed before (PRE) and after (POST) supplementation through a 5-min simulated fight (randori) followed by 3 bouts of the Special Judo Fitness Test (SJFT). Blood samples were collected for blood $\mathrm{pH}$, bicarbonate $\left(\mathrm{HCO}_{3}{ }^{-}\right)$and lactate determination.

Results: Beta-alanine supplementation improved the number of throws per set and the total number of throws (both $p<0.05$ ). Placebo did not change these variables (both $p>0.05$ ). Blood $\mathrm{pH}$ and $\mathrm{HCO}_{3}-$ reduced after exercise (all $p<0.001$ ), with no between-group differences (all $p>0.05$ ). However, the lactate response to exercise increased in the beta-alanine group as compared to placebo $(p<0.05)$.

Conclusions: In conclusion, 4 weeks of beta-alanine supplementation effectively enhance judo-related performance in highly-trained athletes.
\end{abstract}

(C) 2016 Sports Medicine Australia. Published by Elsevier Ltd. All rights reserved.

\section{Introduction}

Judo is an Olympic sport which requires athletes to perform high-intensity efforts interspersed by short recovery periods. ${ }^{1}$ Although a judo match can be finished whenever an ippon (perfect throw) occurs, most fights in elite judo last around 3 min. ${ }^{1}$ Moreover, it is common for an athlete to dispute several fights in the same day. ${ }^{2}$ Altogether, these data indicate that judo is highly intensive in nature. Studies have shown a high demand for the glycolytic metabolism during judo combats, as suggested by the elevated blood lactate levels after simulated and official judo combats. ${ }^{2}$ This suggests that hydrogens cations $\left(\mathrm{H}^{+}\right)$are produced and accumulated at high rates during judo combats, inducing muscle acidosis.

\footnotetext{
* Corresponding author.

E-mail addresses: vitor.painelli@usp.br, vitor_pa@hotmail.com (V. de Salles Painelli).
}

Muscle acidosis has been considered a major cause of fatigue during high-intensity intermittent exercises. Studies have demonstrated that $\mathrm{H}^{+}$may compete with calcium ions for the myosin-binding site, interfering with the contractile processes. ${ }^{3}$ In addition, $\mathrm{H}^{+}$accumulation has been shown to inhibit key-steps of the anaerobic metabolism, such as phosphocreatine resynthesis ${ }^{4}$ and phosphofructokinase activity. ${ }^{5}$ In sports in which technical excellence is decisive for competitive success, fatigue may be particularly deleterious to performance since it may interfere with technical skills ${ }^{6}$ and decision making. ${ }^{7}$ Hence, nutritional strategies aiming at attenuating muscle acidosis have the potential to enhance sports performance. In fact, there is evidence showing that sodium bicarbonate can improve performance in a wide range of acidotic tasks, including judo. ${ }^{8,9}$ Another buffering ergogenic aid that has been receiving attention in recent years is the supplementation of the amino acid beta-alanine (BA).

$\mathrm{BA}$ is a non-essential, non-proteinogenic amino acid that participates in the synthesis of carnosine ( $\beta$-alanyl-L-histidine), a 
dipeptide abundantly found in the skeletal muscle. Due to its $\mathrm{p} K_{\mathrm{a}}$ of 6.8 , carnosine acts as a $\mathrm{H}^{+}$buffer within the muscle $\mathrm{pH}$ transit range. ${ }^{10}$ Carnosine has been shown to improve the sensitivity of the contractile apparatus to calcium, ${ }^{11}$ although the relevance of such effects to the in vivo muscle function still needs further investigation. ${ }^{12}$ Although BA is endogenously synthesized in hepatocytes, ${ }^{10}$ its synthesis rate is low. Therefore, the availability of BA is the rate-limiting factor for carnosine synthesis in skeletal muscle. ${ }^{13}$ Since Harris et al. have shown that chronic BA supplementation increases the intramuscular carnosine content by $\sim 40-80 \%,{ }^{13}$ many investigations have examined the ergogenic effects of BA on exercise capacity and performance. ${ }^{10,14}$ It has been demonstrated that chronic BA supplementation (typically 4-10 weeks) may improve performance in a wide range of sports, especially in those characterized by a marked glycolytic demand. ${ }^{15-22}$ Interestingly, BA appears to be as effective in highly-trained athletes as it is in physically active individuals. ${ }^{23}$ Since BA has shown to be ergogenic in highly-trained athletes and in sports limited by acidosis, we hypothesized that BA could improve judo-related performance. In the present investigation, we evaluated the effects of 4 weeks of BA supplementation on a judo-specific protocol designed to mimic the metabolic demands of judo.

\section{Methods}

Twenty-three well-trained male judo competitors volunteered to participate in this study. All athletes were actively participating in national- and international-level official competitions at the time of data collection. Participants were refrained from creatine or BA supplements for at least 3 and 6 months, respectively, prior to participation. All participants were fully informed of the risks and discomforts before giving their written informed consent. The study was approved by the institution's Ethical Committee.

In this randomised, double-blind, placebo-controlled, parallelgroup trial, athletes were randomly allocated to receive either BA $(n=12$; age $=17 \pm 2 \mathrm{y}$; body mass $=74.2 \pm 11.6 \mathrm{~kg}$; training experience $=9 \pm 3 \mathrm{y}$; training volume $=20 \mathrm{hweek}^{-1}$ ) or placebo (PL, $n=11$; age $=19 \pm 3 \mathrm{y}$; body mass $=71.5 \pm 10.7 \mathrm{~kg}$; training experience $=11 \pm 4 \mathrm{y}$; training volume $=20 \mathrm{~h}_{\text {week }}^{-1}$; all $p>0.05$ vs. BA). Randomisation was conducted in blocks with groups being equalised according to the total throws performed in the Special Judo Fitness Test (SJFT, a validated judo-specific test ${ }^{24}$ ) undertook on the familiarization session. The BA group received $6.4 \mathrm{~g} \mathrm{day}^{-1}$ of beta-alanine (CarnoSyn, Compound Solutions Inc., USA) during 4 weeks. The supplement was provided in acid-resistant hypromellose capsules (DRcaps, Capsugel, USA) in order to slow absorption into the bloodstream and avoid paraesthesia. Athletes took $2 \times 800 \mathrm{mg}$ capsules per dose along with main meals, four times per day. The PL group received the exact same amount of dextrose in capsules identical in number, size and appearance. The efficacy of the blinding procedure was tested by asking the athletes to identify the supplement they believed they had received. Perceived side effects were reported throughout the study.

Athletes underwent the same experimental sessions on 2 different occasions, 4 weeks apart. The trials were completed before (PRE) and after 4 weeks of supplementation (POST). No competition was scheduled at least 14 days before or after each trial, so that no athlete was undergoing or recovering from rapid weight loss during the trials. In each trial, athlete's performance was assessed through three sets of the SJFT preceded by a 5-min simulated judo combat. All experimental sessions occurred at the same time of the day (i.e., 14:00 h) on the same weekday. Participants were requested to abstain from alcohol and unaccustomed exercise in the 48 hours prior to the experimental sessions as well as caffeine in the $16 \mathrm{~h}$ pre- ceding the tests. Participants arrived at the laboratory at least $2 \mathrm{~h}$ following their last meal, and immediately began their warm-up. Ad libitum water consumption was allowed during the sessions.

Athletes warmed up freely for $10 \mathrm{~min}$ as they were used to do before their routine training sessions. Afterwards, they started a randori (judo fight) against an ability- and weight-matched judo athlete, as assigned by their coaches. The randori lasted $5 \mathrm{~min}$, regardless of the occurrence of ippon (which would determine the end of the fight in official competitions), and it was followed by a 10 -min passive recovery period. Athletes then performed 3 bouts of the SJFT interspersed by a 3-min passive recovery period (see Supplementary Fig. S1, Panel A in the online version at DOI: http://dx. doi.org/10.1016/j.jsams.2016.08.014). The test consists of one athlete (TORI) applying a judo technique (ippon seoi nage) to throw two other athletes (UKE) as many times as possible. The TORI begins the test between the two UKE, $3 \mathrm{~m}$ apart from each other. On a signal, the TORI runs and throws one UKE and then runs back to throw the other (see Supplementary Fig. S1, Panel B in the online version at DOI: http://dx.doi.org/10.1016/j.jsams.2016.08.014). Each SJFT comprises three sub-sets (lasting 15 s, 30 s, and 30 s, respectively), interspersed by 10 -s recovery periods each. SJFT performance is determined through the number of throws performed. In this study, athletes performed three bouts of the SJFT, totalising 9 sub-sets. The coefficient of variation (CV) for total throws obtained from the familiarisation and PRE-supplementation session was $3.4 \pm 2.4 \%$ and $3.6 \pm 3.9 \%$ for the PL and BA groups, respectively.

Blood samples were collected after warm-up (baseline), immediately post-randori and after SJFT for the determination of blood $\mathrm{pH}$, blood bicarbonate, base excess and plasma lactate. An additional sample was collected $5 \mathrm{~min}$ after SJFT for plasma lactate analysis. Samples were taken from the antecubital vein using a heparinised syringe (BD A-Line) and immediately injected into an automatized blood gas analyser (Rapid Point $350^{\circledR}$, Siemens) for $\mathrm{pH}$ and $\mathrm{PCO}_{2}$ determination. Bicarbonate was calculated according to the Andersen-Hasselbach equation. A small aliquot of blood $(20 \mu \mathrm{l})$ was placed in a microtube containing the same volume of an ice-cold $2 \% \mathrm{NaF}$ solution and centrifuged at $2000 \times \mathrm{g}$ for $5 \mathrm{~min}$ at $4{ }^{\circ} \mathrm{C}$; plasma was stored at $-80^{\circ} \mathrm{C}$ for further analysis. Plasma lactate was determined spectrophotometrically using an enzymatic-colorimetric method (Katal, Interteck, Sao Paulo, Brazil) in a microplate-based assay using a linear 5-point standard curve to calculate unknown values by interpolation.

To control for intervening variables, food intake was assessed during the supplementation period using three 24-h dietary recalls undertaken on separate days ( 2 weekdays and 1 weekend day), with the aid of a photo album of real-sized foods and portions. Energy and macronutrient intake were analysed with Avanutri software (Sao Paulo, Brazil) and BA intake from food was calculated based on Jones et al. ${ }^{25}$

Data are presented as mean \pm SD and were analysed using SAS v.9.3. Unpaired $t$ tests were used to compare performance, food intake, and participant characteristics between groups at the presupplementation period. Mixed models were used to examine the effect of supplementation on the dependent variables, assuming "group" (BA and PL) and "time" (PRE, POST and multiple analysis within the same experimental session) as fixed factors, and "athletes" as a random factor. The Tukey-Kramer adjustment was used for multiple comparisons. Unpaired t tests were used to compare the absolute changes between groups for total throws, and effect sizes were calculated according to Cohen's D. The smallest worthwhile change for the total number of throws between the PRE and POST trials was also determined for both groups, as previously described. ${ }^{20,22}$ The Fischer exact test was used to compare the proportion of participants who correctly guessed their allocation in the group. Statistical significance was accepted at $P \leq 0.05$. 
A
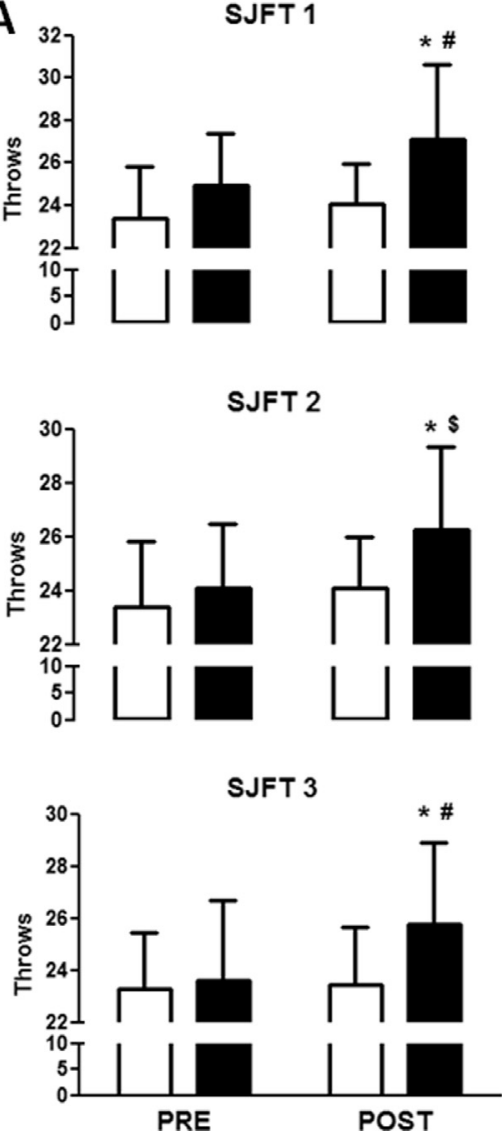

B
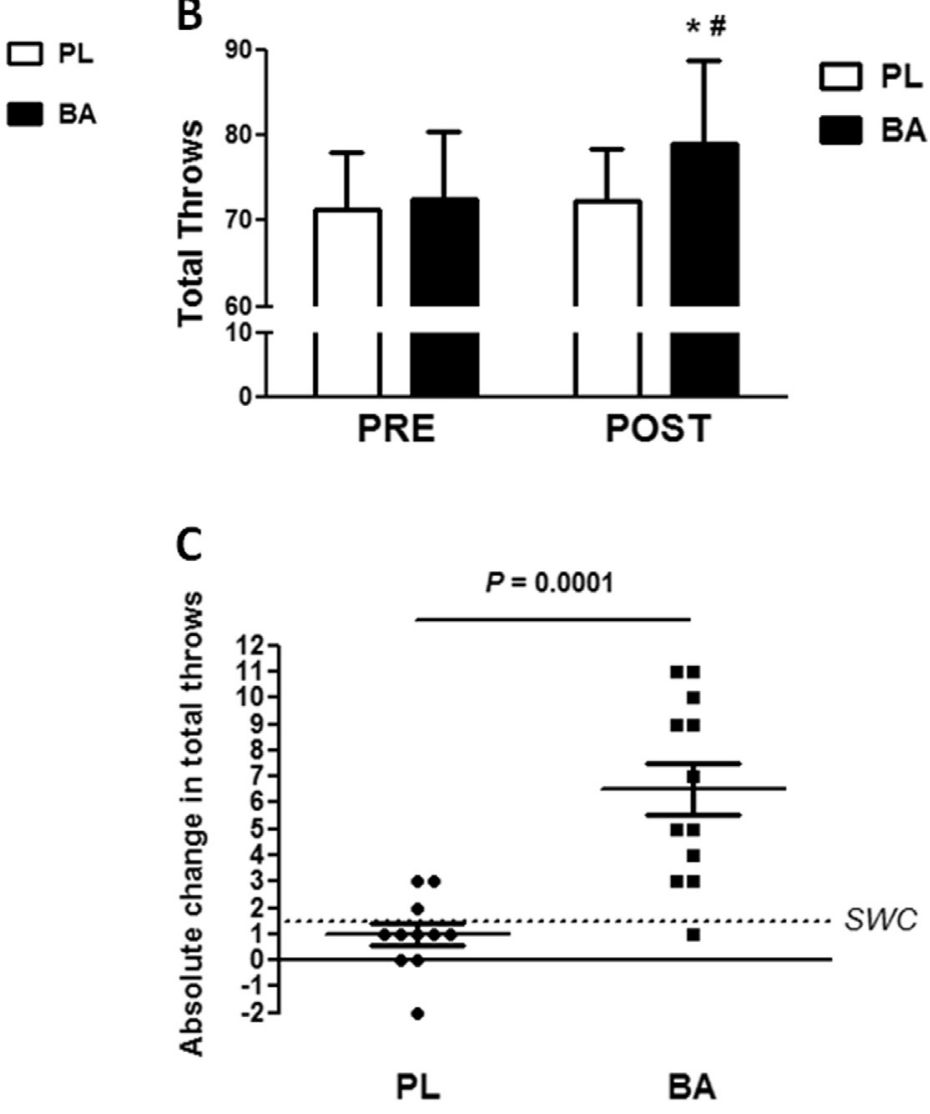

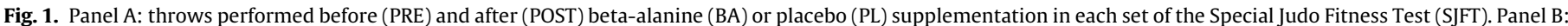

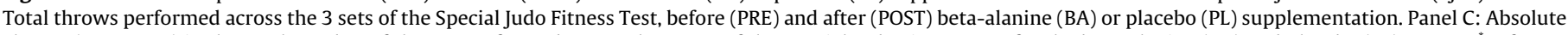

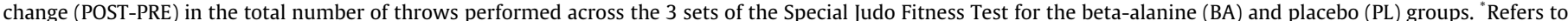

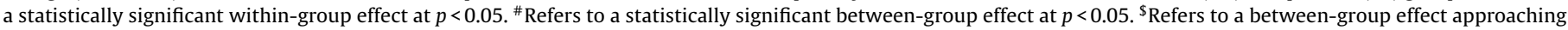
significance $(p=0.053)$. The dotted line refers to the smallest worthwhile change (SWC).

\section{Results}

At PRE, the number of throws significantly decreased from the SJFT1 to the SJFT2 and SJFT3 (for both groups, $p<0.001$ ), with no significant difference between the groups (SFT1, $p=0.8$; SFT2, $p=0.516$; and SFT3, $p=0.779$ ). A significant interaction effect on the number of throws per set $(F=8.25 ; p<0.001)$ was observed. Posthoc analysis showed a within-group effect for BA in every bout of the SJFT (all $p<0.001$; Fig. 1, Panel A). In contrast, the number of throws per set in PL did not change from PRE to POST in any of the SJFT bouts (all $p>0.05$; Fig. 1, Panel A). Furthermore, at POST, the number of throws for the BA group was significantly higher (or approached significance) in all SJFT bouts in comparison to PL (SFT1, $p=0.035$; SFT2, $p=0.053$; and SFT3, $p=0.040$ ).

No between-group differences $(p=0.669)$ were observed at PRE for the total number of throws. A significant interaction effect on the total number of throws was observed $(F=24.14$; $p<0.001$ ) (Fig. 1, Panel B). Analysis revealed a significant within$(p<0.001 ; 95 \% \mathrm{CI}=-1.0$ to $14.0 ; \mathrm{ES}=0.77)$ and between-group effect ( $p=0.046 ; 95 \% \mathrm{CI}=-0.2$ to 13.8 ; $\mathrm{ES}=0.88$ ) for BA. No withingroup effect was observed for PL $(p=0.229 ; 95 \% \mathrm{CI}=-4.7$ to 6.7 ; $\mathrm{ES}=0.17$ ) (Fig. 1, Panel B). The absolute increase in the total number of throws from PRE to POST in BA group was significantly different from that in PL $(p<0.001)$ (Fig. 1, Panel C). Individual analysis showed that all the 12 athletes improved SJFT performance after BA supplementation (change: $+8.9 \pm 4.7 \%$ ), with 9 improving above the $\mathrm{CV}$. Although 8 of 11 increased the total number of throws with $\mathrm{PL}$, none of them improved above the CV (change: $+1.5 \pm 2.0 \%$ ).
Similarly, 11 out 12 athletes improved the total number of throws after BA supplementation by a magnitude greater than the smallest worthwhile change. On the other hand, only 3 of 11 improved SJFT performance after PL supplementation by a magnitude greater than the smallest worthwhile change (Fig. 1, Panel C).

No significant differences were observed in any of the blood variables between the experimental conditions at PRE. Blood $\mathrm{pH}$ significantly reduced from Baseline to Post-Randori and Post-SJFT for both groups (both $p<0.001$ ), but without any interaction effect $(F=0.59 ; p=0.710$ ) (Table 1$)$. Similarly, blood $\mathrm{HCO}_{3}{ }^{-}$and base excess were significantly reduced after the exercise (Post-Randori and Post-SJFT compared to Baseline) in both groups (both $p<0.001$ ), with no influence of supplementation (Table 1). Plasma lactate significantly increased from Baseline to Post-Randori and Post-SJFT for both groups (both $p<0.001$ ) with a significant interaction effect being observed $(F=2.97 ; p=0.024)$. A significant lactate increase $(p=0.001)$ was observed for BA at Post-SJFT compared to PRE (Fig. 2).

The average daily intake of energy (BA: $2201 \pm 121 \mathrm{kcal}$; PL: $2275 \pm 147 \mathrm{kcal} ; \quad p=0.204$ ), carbohydrate (BA: $54.3 \pm 3.3 \%$; PL: $55.1 \pm 2.2 \% ; p=0.469$ ), lipid (BA: $27.9 \pm 2.5 \%$; PL: $27.2 \pm 2.7 \%$; $p=0.524$ ), protein (BA: $17.8 \pm 1.9 \%$; PL: $17.6 \pm 1.8 \% ; p=0.837$ ) or BA (BA: $542.5 \pm 99.9 \mathrm{mg}$; PL: $539.6 \pm 108.2 \mathrm{mg} ; p=0.946$ ) did not significantly differ between the groups across the study period.

Only 1 of 12 participants reported mild paraesthesia with BA supplementation. Curiously, 1 participant from the PL group also reported mild paraesthesia. No other side effects were reported. Only 6 out of 11 participants successfully predicted they were 
Table 1

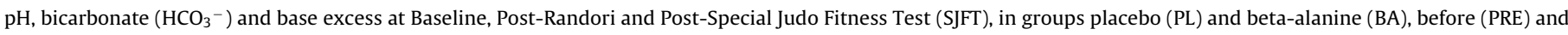
after (POST) the supplementation.

\begin{tabular}{|c|c|c|c|c|c|c|}
\hline & \multicolumn{3}{|l|}{$\mathrm{PL}$} & \multicolumn{3}{|l|}{$\mathrm{BA}$} \\
\hline & Baseline & Post-Randori & Post-SJFT & Baseline & Post-Randori & Post-SJFT \\
\hline \multicolumn{7}{|l|}{$\mathrm{pH}$} \\
\hline PRE & $7.31 \pm 0.05$ & $7.12 \pm 0.08^{*}$ & $7.13 \pm 0.08^{*}$ & $7.31 \pm 0.05$ & $7.09 \pm 0.05^{*}$ & $7.16 \pm 0.06^{*}$ \\
\hline POST & $7.33 \pm 0.03$ & $7.14 \pm 0.11^{*}$ & $7.18 \pm 0.05^{*}$ & $7.33 \pm 0.04$ & $7.12 \pm 0.10^{*}$ & $7.14 \pm 0.08^{*}$ \\
\hline \multicolumn{7}{|c|}{$\mathrm{HCO}_{3}{ }^{-}(\mathrm{mmol} / \mathrm{L})$} \\
\hline PRE & $26.23 \pm 1.29$ & $22.33 \pm 1.74^{*}$ & $17.52 \pm 3.00$ & $27.00 \pm 1.77$ & $19.70 \pm 3.28^{*}$ & $16.08 \pm 2.79^{*}$ \\
\hline POST & $27.40 \pm 1.36$ & $21.36 \pm 2.96^{*}$ & $17.81 \pm 2.28^{*}$ & $27.21 \pm 1.75$ & $22.80 \pm 3.08^{*}$ & $15.27 \pm 3.37^{*}$ \\
\hline \multicolumn{7}{|c|}{ Base excess $(\mathrm{mmol} / \mathrm{L})$} \\
\hline PRE & $-1.38 \pm 1.25$ & $-8.98 \pm 2.33^{*}$ & $-12.16 \pm 3.31^{*}$ & $-0.54 \pm 1.46$ & $-9.66 \pm 4.15^{*}$ & $-12.01 \pm 2.75^{*}$ \\
\hline POST & $-0.62 \pm 0.47$ & $-10.44 \pm 2.65^{*}$ & $-11.33 \pm 3.08^{*}$ & $-0.17 \pm 1.49$ & $-8.96 \pm 4.36^{*}$ & $-13.81 \pm 4.30^{*}$ \\
\hline
\end{tabular}

Means a significant difference from Baseline within the same time at $p \leq 0.05$.

Means a significant difference from Post-Randori within the same time at $p \leq 0.05$. The data are expressed as mean $\pm S D$.
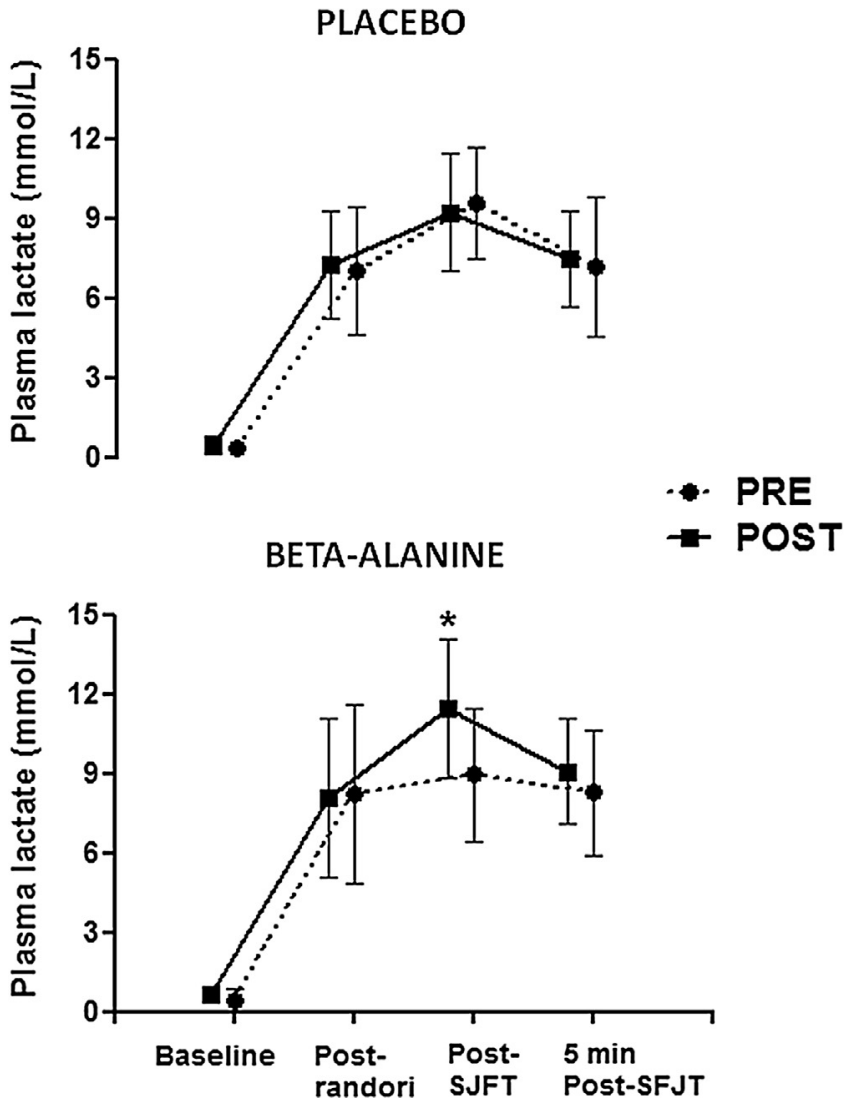

Fig. 2. Plasma lactate concentration at Baseline, Post-Randori, Post-SJFT and $5 \mathrm{~min}$ (min) Post-Randori, before and after placebo (PL) or beta-alanine (BA) supplementation. Legend: The circles with dotted lines refer to plasma lactate values before (PRE) the supplementation, while the squares with solid lines refer to plasma lactate values after the supplementation (POST). The symbol ${ }^{*}$ refers to a significant within-group effect at $p<0.05$.

ingesting placebo, while 5 out of 12 successfully identified they were ingesting BA. There were no significant differences between groups for supplements identification (Fisher Exact Test: $p=0.684$ ).

\section{Discussion}

This study aimed to examine the ergogenic effects of BA supplementation on judo-related performance. Given the intense and acidotic nature of judo and judo-related tasks, ${ }^{24}$ confirmed in our study by the fall in blood $\mathrm{pH}$ following the tests, we hypothesized that performance in a judo-specific protocol could benefit from BA. In line with our hypothesis, the main findings of this inves- tigation indicated that BA supplementation significantly improved performance in highly-trained judo athletes.

Some studies have failed to observe positive effects of BA on sports tasks, such as 400-m sprint running, ${ }^{26} 4-\mathrm{min}^{2728}$ and 1 -h cycling time-trials. ${ }^{29}$ In contrast, positive effects of BA have been shown in sports such as cycling (e.g., sprints, 4-km time-trial), ${ }^{16,22}$ boxing, ${ }^{17}$ soccer, ${ }^{18} 100-\mathrm{m}$ and $200-\mathrm{m}$ freestyle swimming timetrial $^{19}, 800-\mathrm{m}$ running ${ }^{20}$ and $2000-\mathrm{m}$ rowing time-trials ${ }^{21}$. One possible explanation for the lack of positive results in some studies $^{26-29}$ may rely on the type of the exercise tests, which may have been too short or too long to properly stress the anaerobic glycolytic metabolism, and hence, induce $\mathrm{H}^{+}$accumulation. Therefore, one could speculate that performance in high-intensity, short-duration exercises would be more prone to be improved by buffering agents. In support of this notion, a meta-analysis showed that exercise tasks lasting 1-4 min were greatly improved by BA when compared with those lasting less or longer than that. ${ }^{14}$ The results from the current investigation reinforce the ergogenic properties of BA supplementation in acidotic tasks.

Although there is evidence showing ergogenic effects of BA regardless of the individual's training status, ${ }^{23}$ studies enrolling highly-trained athletes are important to confirm the efficacy of a nutritional strategy in sports settings. Nevertheless, such studies are limited due to several methodological issues, including small groups of elite athletes, complex competition and training schedules, unavailability to undertake invasive assessments, and the typical use of a multitude of supplements by these athletes. In this regard, the current investigation adds to literature by examining the effects of BA supplementation in highly-trained, elite judo competitors. In our study, the total number of throws did not differ between the PL and BA groups at PRE, indicating that our randomisation successfully generated similar groups. Notwithstanding, 4 weeks of BA promoted a $~ 9 \%$ improvement in total throws with 11 out 12 improving above the smallest worthwhile changes in performance.

Studies with supplements capable of increasing buffering capacity (e.g., BA and sodium bicarbonate) have consistently shown that the ergogenic effects are particularly evident when acidosis is already installed, such as in the final bouts of an intermittent exercise protocol. ${ }^{8,9,30}$ However, a significant improvement was observed in all three SJFT bouts in the present investigation. This can be explained by the residual fatigue (acidosis) imposed by the randori preceding the SJFT bouts, which was included in the testing protocol in an attempt to mimic the multiple fights that judo athletes undertake in actual competitions. The present data seem to concur with a previous study showing the beneficial effects of BA supplementation in judo athletes tested in a laboratorial environment. ${ }^{30}$ 
Overall, the current study also reinforces the potential ergogenic effects of buffering agents in judo performance. Artioli et al. ${ }^{8}$ observed a significant increase in the total number of throws after 3 bouts of the SJFT following acute sodium bicarbonate supplementation in well-trained judo athletes. Recently, Felippe et al. ${ }^{9}$ demonstrated that, in the third bout of the SJFT, sodium bicarbonate plus caffeine resulted in a similar increase in the number of throws compared to sodium bicarbonate alone, suggesting that this improvement occurred mainly due to the sodium bicarbonate effect. In agreement, our results allow us to conclude that BA is also an effective nutritional strategy to improve judo-related performance. Although studies on the combination of different buffering agents exist, ${ }^{31}$ future studies are warranted to examine which one of these interventions may elicit the greatest improvement in judo performance, and, more importantly, whether their combination would have additive ergogenic properties.

The main limitation of this study was the lack of muscle biopsies to confirm the efficacy of BA in increasing the muscle carnosine content. However, to our knowledge, all the studies so far using BA doses varying from 1.6 to $6.4 \mathrm{~g} \mathrm{~d}^{-1}$ for 4 weeks or longer have consistently reported increases of at least $8 \mathrm{mmol} \mathrm{kg}^{-1}$ dry muscle of intramuscular carnosine, corresponding to an increase of $40 \% .{ }^{10,14}$ According to estimates by Harris et al., a $40 \%$ increase in muscle carnosine would represent a $\sim 4 \%$ increase in whole muscle buffering capacity and a $\sim 7 \%$ in type II fibres buffering capacity. ${ }^{13}$ The increased blood lactate response to exercise with BA is an indirect evidence of the increased buffering capacity. Based on these data, it seems likely that our BA supplementation protocol resulted in increased muscle carnosine with a consequential increase in intramuscular buffering capacity.

In accordance with previous studies examining the effects of BA on blood gas parameters, ${ }^{1521}$ our results showed no effects of supplementation on blood $\mathrm{pH}$. Since carnosine is a cytoplasmic dipeptide, ${ }^{10,13}$ a direct effect on blood $\mathrm{pH}$ would not be expected. However, since athletes taking BA performed more throws and presented higher lactate responses, energy transfer via glycolysis was likely increased, and a parallel increase in $\mathrm{H}^{+}$could be expected. The lack of changes in blood $\mathrm{pH}$ in the BA group, however, suggests that any extra $\mathrm{H}^{+}$produced in the muscle did not reach the bloodstream, being probably neutralised inside the cells.

The only known side effect of BA supplementation is paraesthesia. Symptoms typically appear within $\sim 30$ min when doses larger than $800 \mathrm{mg}$ of BA in powder are ingested, suggesting that paraesthesia is related to elevated BA levels in blood. ${ }^{13}$ These symptoms are not observed when $1600 \mathrm{mg}$ of BA are provided in controlled-release tablets. ${ }^{32}$ In the current investigation, only 1 athlete reported paraesthesia, indicating that the strategy adopted in our study to slow the absorption of BA was successful in preventing paraesthesia. Moreover, our athletes were not able to correctly guess the substance that they were ingesting, indicating that the blinding of our study remained intact.

\section{Conclusion}

To conclude, 4 weeks of BA supplementation effectively improved judo-related performance in well-trained judo athletes, suggesting that this modality can also benefit from the ergogenic effects of BA.

\section{Practical implications}

- BA supplementation is an effective dietary intervention capable of enhancing judo-related performance.
- Apart from physically active individuals, well-trained athletes in high-intensity intermittent sports can also benefit from the ergogenic effects of BA.

- The use of acid-resistant hypromellose capsules seems to effectively prevent BA-induced paraesthesia.

\section{Acknowledgements}

The authors are grateful to all the athletes and their coaches for their volunteer efforts to take part in the study. The authors are also thankful to Sao Paulo Research Foundation (FAPESP grants number: 2013/04806-0, 2013/14746-4 and 2014/11948-8) for the financial support. The authors declare they have no conflict of interest.

\section{References}

1. Franchini E, Artioli GG, Brito CJ. Judo combat: time-motion analysis and physiology. Int J Perform Anal Sport 2013; 13:624-641.

2. Artioli GG, Iglesias RT, Franchini E et al. Rapid weight loss followed by recovery time does not affect judo-related performance. J Sports Sci 2010; 28(1):21-32.

3. Fabiato A, Fabiato F. Effects of $\mathrm{pH}$ on the myofilaments and the sarcoplasmic reticulum of skinned cells from cardiace and skeletal muscles. J Physiol 1978; 276:233-255

4. Harris RC, Edwards RH, Hultman E et al. The time course of phosphorylcreatine resynthesis during recovery of the quadriceps muscle in man. Pflugers Arch 1976; 367(2):137-142.

5. Sutton JR, Jones NL, Toews CJ. Effect of PH on muscle glycolysis during exercise. Clin Sci (Lond) 1981; 61(3):331-338.

6. Gabett TJ. Influence of fatigue on tackling technique in rugby league players. $J$ Strength Cond Res 2008; 22(2):625-632.

7. De Lima EL, Tortoza C, Laureano LC et al. Study of the correlation between the velocity of motor reaction and blood lactate in different times of combat in judo. Rev Bras Med Esporte 2004; 10(5):339-343.

8. Artioli GG, Gualano B, Coelho DF et al. Does sodium-bicarbonate ingestion improve simulated judo performance? Int J Sport Nutr Exerc Metab 2007; 17(2):206-217.

9. Felippe LC, Lopes-Silva JP, Bertuzzi R et al. Separate and combined effects of caffeine and sodium bicarbonate intake on judo performance. Int J Sports Physiol Perform 2015. http://dx.doi.org/10.1123/ijspp.2015-0020.

10. Artioli GG, Gualano B, Smith A et al. Role of beta-alanine supplementation on muscle carnosine and exercise performance. Med Sci Sports Exerc 2010; 42(6):1162-1173.

11. Dutka TL, Lamboley CR, McKenna MJ et al. Effects of carnosine on contractile apparatus $\mathrm{Ca}^{2}+$ sensitivity and sarcoplasmic reticulum $\mathrm{Ca}^{2}+$ release in human skeletal muscle fibers. J Appl Physiol 2012; 112(5):728-736.

12. Hannah R, Stannard RL, Minshull C et al. $\beta$-Alanine supplementation enhances human skeletal muscle relaxation speed but not force production capacity. J Appl Physiol 2015; 118(5):604-612.

13. Harris RC, Tallon MJ, Dunnett M et al. The absorption of orally supplied betaalanine and its effect on muscle carnosine synthesis in human vastus lateralis. Amino Acids 2006; 30(3):279-289.

14. Hobson RM, Saunders B, Ball G et al. Effects of $\beta$-alanine supplementation on exercise performance: a meta-analysis. Amino Acids 2012; 43(1):25-37.

15. Baguet A, Koppo K, Pottier A et al. Beta-alanine supplementation reduces acidosis but not oxygen uptake response during high-intensity cycling exercise. Eur J Appl Physiol 2010; 108(3):495-503.

16. Van Thienen R, Van Proeyen K, Vanden Eynde B et al. Beta-alanine improves sprint performance in endurance cycling. Med Sci Sports Exerc 2009; 41(4):898-903.

17. Donovan T, Ballam T, Morton JP et al. $\beta$-Alanine improves punch force and frequency in amateur boxers during a simulated contest. Int J Sport Nutr Exerc Metab 2012; 22(5):331-337.

18. Saunders B, Sunderland C, Harris RC et al. $\beta$-alanine supplementation improves YoYo intermittent recovery test performance. J Int Soc Sports Nutr 2012; 9(1):39.

19. De Salles Painelli V, Roschel H, Jesus F et al. The ergogenic effect of beta-alanine combined with sodium bicarbonate on high-intensity swimming performance. Appl Physiol Nutr Metab 2013; 38(5):525-532.

20. Ducker KJ, Dawson B, Wallman KE. Effect of beta-alanine supplementation on 800-m running performance. Int J Sport Nutr Exerc Metab 2013; 23(6):554-561.

21. Hobson RM, Harris RC, Martin D et al. Effect of beta-alanine, with and without sodium bicarbonate, on 2000-m rowing performance. Int J Sport Nutr Exerc Metab 2013; 23(5):480-487.

22. Belliger PM, Minahan CL. The effect of $\beta$-alanine supplementation on cycling time trials of different length. Eur J Sport Sci 2015; 11:1-8.

23. De Salles Painelli V, Saunders B, Sale C et al. Influence of training status on highintensity intermittent performance in response to $\beta$-alanine supplementation. Amino Acids 2014; 46(5):1207-1215.

24. Franchini E, Sterkowicz S, Szmatlan-Gabrys U et al. Energy system contributions to the special judo fitness test. Int J Sports Physiol Perform 2011; 6(3):334-343.

25. Jones G, Smith M, Harris R. Imidazole dipeptide content of dietary sources commonly consumed within the British diet. Proc Nutr Soc 2011; 70(6):363. 
26. Derave W, Ozdemir MS, Harris RC et al. Beta-Alanine supplementation augments muscle carnosine content and attenuates fatigue during repeated isokinetic contraction bouts in trained sprinters. J Appl Physiol 2007; 103(5): 1736-1743.

27. Bellinger PM, Howe ST, Shing CM et al. The effect of combined $\beta$-alanine and $\mathrm{NaHCO}_{3}$ supplementation on cycling performance. Med Sci Sports Exerc 2012; 44(8):1545-1551.

28. Howe ST, Bellinger PM, Driller MW et al. The effect of $\beta$-alanine supplementation on isokinetic force and cycling performance in highly-trained cyclists. Int J Sport Nutr Exerc Metab 2014; 23(6):562-570.
29. Chung W, Baguet A, Bex T et al. Doubling of muscle carnosine concentration does not improve laboratory 1-h cycling time trial performance. Int J Sport Nutr Exerc Metab 2014; 24(3):315-324.

30. Tobias G, Benatti FB, de Salles Painelli V et al. Additive effects of beta-alanine and sodium bicarbonate on upper-body intermittent performance. Amino Acids 2013; 45(2):309-317.

31. Naderi A, Earnest CP, Lowery RP et al. Co-ingestion of nutritional ergogenic aids and high-intensity exercise performance. Sports Med 2016 [in press].

32. Decombaz J, Beaumont M, Vuichoud J et al. Effect of slow-release beta-alanine tablets on absorption kinetics and paresthesia. Amino Acids 2011; 43(1):67-76 